09,11

\title{
Soft mode mechanism of the transition into the low-temperature ferroelastic phase in $\mathrm{K}_{3} \mathrm{Na}\left(\mathrm{CrO}_{4}\right)_{2}$ crystals
}

\author{
(c) Yu.E. Kitaev ${ }^{1}$, A.S. Krylov ${ }^{2}$, T.I. Maksimova ${ }^{1}$ \\ ${ }^{1}$ loffe Institute, \\ St. Petersburg, Russia \\ ${ }^{2}$ Kirensky Institute of Physics, Siberian Branch of the Russian Academy of Sciences, \\ Krasnoyarsk, Russia \\ E-mail: yu.kitaev@mail.ru
}

(Received May 18, 2016).

The sequence of phase transitions $P \overline{3} m 1 \rightarrow C 2 / m \rightarrow C 2 / c$ in $\mathrm{K}_{3} \mathrm{Na}\left(\mathrm{CrO}_{4}\right)_{2}$ crystals and existence of the intermediate phase have been studied both experimentally by the Raman scattering method and theoretically by programs and retrieval tools of the Bilbao Crystallographic Server. A zone-edge soft mode responsible for a transition from the intermediate $C 2 / \mathrm{m}$ to a low-symmetry $C 2 / c$ phase at $T=210 \mathrm{~K}$ has been discovered experimentally for the first time. The symmetry of the soft mode has been established theoretically to be $A_{2}^{+}$. The temperature interval of the intermediate monoclinic phase has been determined to be $239-210 \mathrm{~K}$.

This work was supported by a grant from the Department of Physical Sciences of the Russian Academy of Sciences within the framework of the program „Basic Optical Spectroscopy and Its Applications.“

\section{Introduction}

The $\mathrm{K}_{3} \mathrm{Na}\left(\mathrm{CrO}_{4}\right)_{2}$ crystals belong to the glaserite family of ferroelastic crystals and have the trigonal phase $P \overline{3} \mathrm{~m} 1$ above $239 \mathrm{~K}$ and the monoclinic phase $C 2 / c$ at lower temperatures as was determined by $X$-Ray measurements in $[1,2]$. In the most studied compound $\mathrm{K}_{3} \mathrm{Na}\left(\mathrm{SeO}_{4}\right)_{2}$ of this family, an intermediate phase has been observed between the paraelectric $P \overline{3} m 1$ and the ferroelastic phase $C 2 / c$ [3-5]. Its symmetry was determined to be $\overline{3}$ in [3] and $P \overline{3} c 1$ in [4]. 4. However, in [5], another space group $B 2 / m$ has been determined to describe the symmetry of the intermediate phase in $\mathrm{K}_{3} \mathrm{Na}\left(\mathrm{SeO}_{4}\right)_{2}$ crystals. Thus, the conclusions on the nature of an intermediate phase in glaserites still remain controversial.

Our recent Raman and infrared studies [6] of $\mathrm{K}_{3} \mathrm{Na}\left(\mathrm{CrO}_{4}\right)_{2}$ crystals in the frequency range of internal $\mathrm{CrO}_{4}$ vibrations $\left(300-1000 \mathrm{~cm}^{-1}\right)$ revealed the existence of two consecutive phase transitions and an intermediate monoclinic phase $C 2 / \mathrm{m}$ in these materials. In the Raman and infrared spectra [6] at temperatures below $200 \mathrm{~K}$, we have observed the splitting of the symmetric bending vibration $v_{2}$ $\left(346 \mathrm{~cm}^{-1}\right)$ and the asymmetric stretching vibration $v_{3}$ $\left(861 \mathrm{~cm}^{-1}\right)$ lines corresponding to internal modes of $\mathrm{CrO}_{4}$ complexes. However, the internal modes of $\mathrm{CrO}_{4}$ rigid units are less sensitive to lattice distortions than the intermolecular low-frequency modes. Therefore, the temperature behavior of the internal modes does not allow the precise determination of the temperature interval of the intermediate phase. Hence, in the present paper we give the result of Raman scattering experiments of $\mathrm{K}_{3} \mathrm{Na}\left(\mathrm{CrO}_{4}\right)_{2}$ in the frequency range of intermolecular vibration to find soft photon modes responsible for phase transitions. We also perform the group theory analysis with the programs and retrieval tools of the Bilbao Crystallographic Server (BCS) [7-12] to establish the symmetry of the soft mode and the contribution on different modes into the global distortion of the cryctal lattice.

\section{Experiments}

The Raman spectra in the backscattering geometry were recorded with a Horiba Jobin Yvon T64000 triple spectrometer equipped with a liquid nitrogen cooled charge coupled device detection system in subtractive dispersion mode. $\mathrm{Ar}^{+}$ion laser Spectra-Physics Stabilite 2017 with $\lambda=514.5 \mathrm{~nm}$ and power $7 \mathrm{~mW}$ on a sample was used as an excitation light source.

Temperature measurements were carried out with closed cycle ARS CS204-X1.SS helium cryostat in the temperature range $8-296 \mathrm{~K}$. The temperature was monitored by LakeShore DT-6SD1.4L silicon diode. During experiments the cryostat was evacuated to $10^{-6}$ mbar. To investigate the low-wavenumber spectra, spectroscopic measurements were performed in the subtractive dispersion mode, which attained a low-wavenumber limit of $6 \mathrm{~cm}^{-1}$ in the present setup. The deformation of the low-wavenumber spectral edge by an optical slit, which sometimes smears the true features of low-wavenumber spectra, was carefully eliminated by rigorous optical alignment. CCD pixel coverage in additive dispersion mode was as fine as $0.3 \mathrm{~cm}^{-1}$ but it was limited by spectrometer spectral resolution of $1.8 \mathrm{~cm}^{-1}$.

The temperature experiments were carried out in the dynamic regime by varying the sample temperature. The rate of temperature variation was $0.5 \mathrm{~K} / \mathrm{min}$. The uncertainty of the measured temperature for a given rate can be estimated as a difference between adjacent measurements and it was $\pm 0.12 \mathrm{~K}$ in one spectrum measurement. Overall time for a single spectrum accumulation was within $30 \mathrm{~s}$. The spectra were acquired with a temperature step $0.25 \mathrm{~K}$. 
Experimental structure data [1,2] of trigonal and monoclinic phases of $\mathrm{K}_{3} \mathrm{Na}\left(\mathrm{CrO}_{4}\right)_{2}$

\begin{tabular}{|c|c|c|c|c|c|c|c|}
\hline \multicolumn{4}{|c|}{ Trigonal phase } & \multicolumn{4}{|c|}{ Monoclinic phase* } \\
\hline \multicolumn{4}{|c|}{$\begin{array}{l}\text { Space group } P \overline{3} m 1(\# 164) \\
a=5.857 \AA \\
b=5.857 \AA \\
c=7.521 \AA \\
\alpha=90^{\circ} \\
\beta=90^{\circ} \\
\gamma=120^{\circ}\end{array}$} & \multicolumn{4}{|c|}{$\begin{array}{l}\text { Space group } C 2 / c(\# 15) \\
a=10.128 \AA \\
b=5.8437 \AA \\
c=15.022 \AA \\
\alpha=90^{\circ} \\
\beta=89.97^{\circ} \\
\gamma=90^{\circ}\end{array}$} \\
\hline $\mathrm{Na} 1 a$ & 0.000000 & 0.000000 & 0.000000 & $\mathrm{Na} 4 a$ & 0.000000 & 0.000000 & 0.000000 \\
\hline $\mathrm{K} 1 b$ & 0.000000 & 0.000000 & 0.500000 & $\mathrm{~K} 4 e$ & 0.000000 & 0.983600 & 0.250000 \\
\hline $\mathrm{K} 2 d$ & 0.333333 & 0.666667 & 0.832600 & $\mathrm{~K} 8 f$ & 0.167100 & 0.494460 & 0.416690 \\
\hline $\operatorname{Cr} 2 d$ & 0.333333 & 0.666667 & 0.273440 & $\mathrm{Cr} 8 f$ & 0.166300 & 0.495280 & 0.136960 \\
\hline $\mathrm{O} 2 d$ & 0.333333 & 0.666667 & 0.487700 & $\mathrm{O} 8 f$ & 0.166000 & 0.513300 & 0.244100 \\
\hline \multirow[t]{3}{*}{$\mathrm{O} 6 i$} & 0.181360 & 0.362720 & 0.197200 & O $8 f$ & 0.089000 & 0.719000 & 0.094300 \\
\hline & & & & $\mathrm{O} 8 f$ & 0.089100 & 0.261000 & 0.104400 \\
\hline & & & & O $8 f$ & 0.317000 & 0.490500 & 0.097000 \\
\hline
\end{tabular}

Footnote. * The low-symmetry structure corresponds to the space group setting with the unique axis $\mathbf{b}$.

This measurement protocol was the same as described in $[13,14]$. The temperature evolution of the Raman spectra in the temperature range of $8-296 \mathrm{~K}$ is presented in Fig. 1. It is clearly seen that the line $33.5 \mathrm{~cm}^{-1}$ at $8 \mathrm{~K}$ tends to

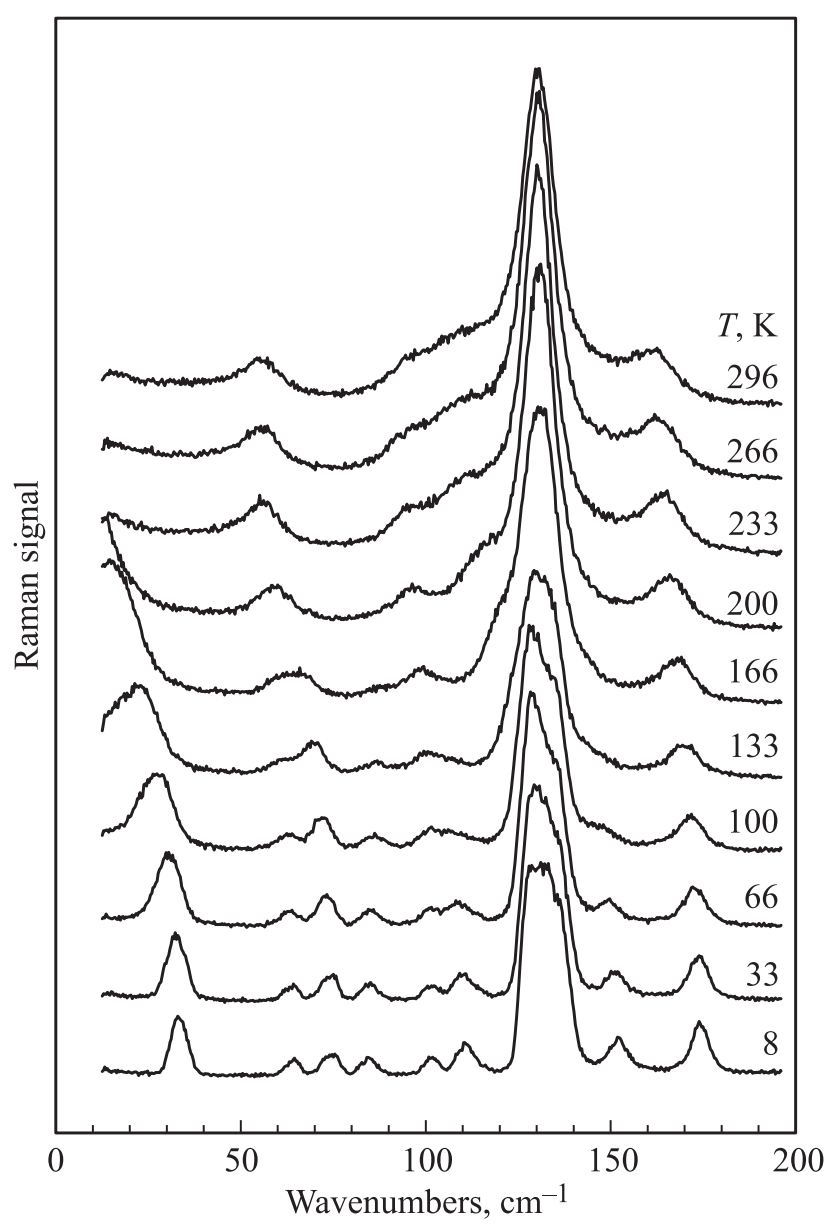

Figure 1. Raman spectra of $\mathrm{K}_{3} \mathrm{Na}\left(\mathrm{CrO}_{4}\right)_{2}$.

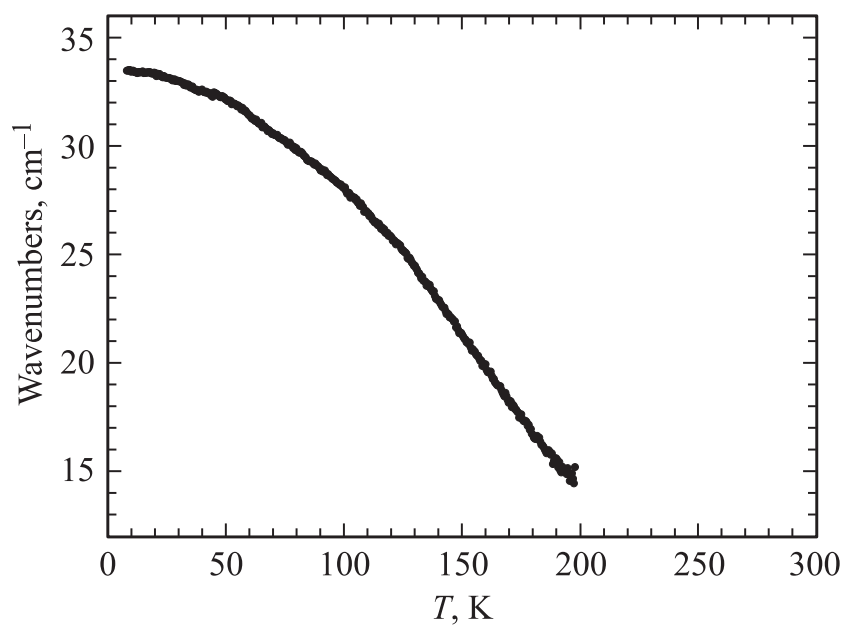

Figure 2. Temperature dependence of the soft mode frequency.

zero when approaching the phase transition temperature at $210 \mathrm{~K}$. The temperature dependence of the soft mode frequency is drawn in Fig. 2.

Above $210 \mathrm{~K}$, no soft mode lines were observed in the first-order Raman spectra. To establish the symmetry of the soft mode and the sequence of phase transitions we carried out theory consideration based on the programs of BCS [7-12].

\section{Theory and discussion}

The programs of BCS [7-12] offer effective tools for group theory analysis of displacive phase transitions in solids.

To analyze the phase transitions in $\mathrm{K}_{3} \mathrm{Na}\left(\mathrm{CrO}_{4}\right)_{2}$ crystals we use the AMPLIMODES program $[10,11]$ which allows one to determine the global structural distortion that relates 


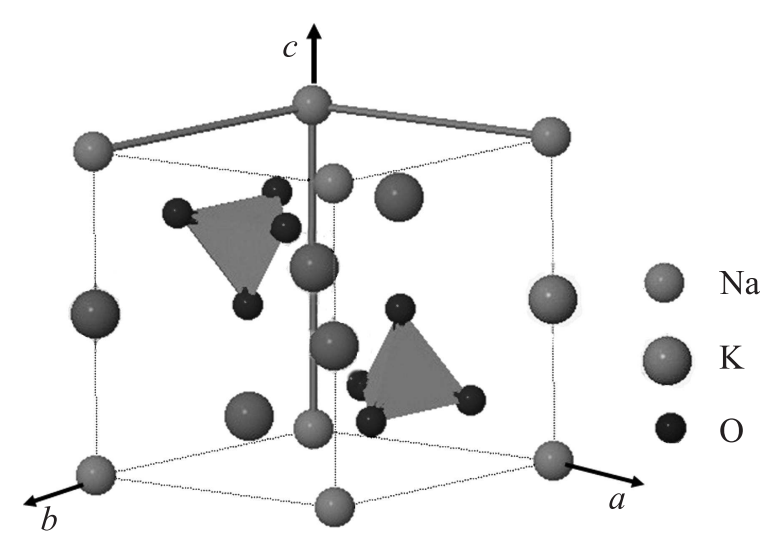

Figure 3. The crystallographic unit cell of the high-temperature trigonal phase of $\mathrm{K}_{3} \mathrm{Na}\left(\mathrm{CrO}_{4}\right)_{2}$. $\mathrm{Cr}$ atoms are inside oxygen tetrahedra.

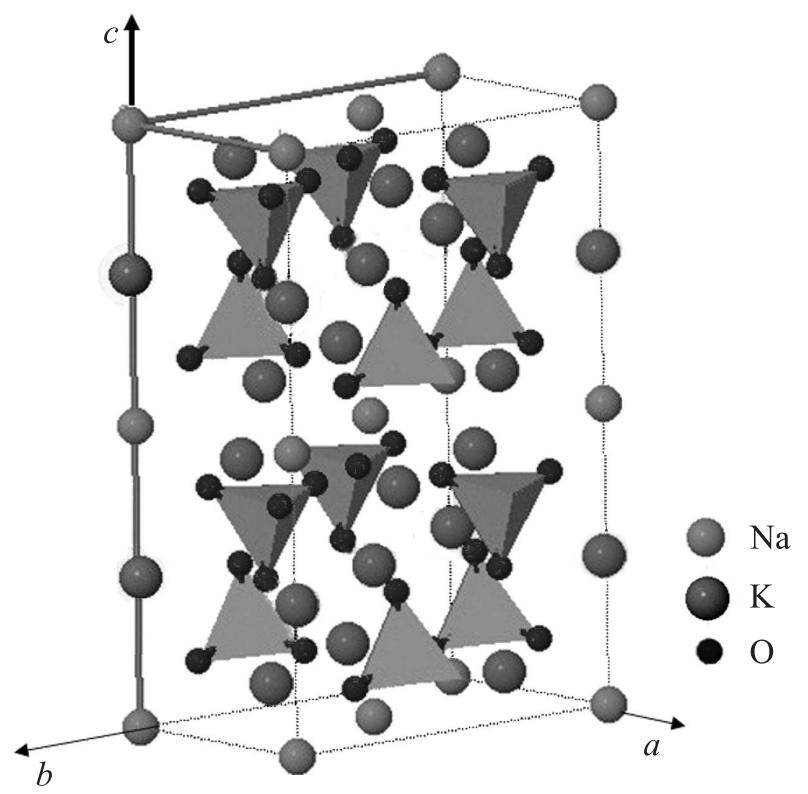

Figure 4. The crystallographic unit cell of the low-temperature monoclinic phase of $\mathrm{K}_{3} \mathrm{Na}\left(\mathrm{CrO}_{4}\right)_{2}$. $\mathrm{Cr}$ atoms are inside oxygen tetrahedra.

\begin{tabular}{|l|c|l|}
\hline \multicolumn{3}{|c|}{ Symmetry modes summary } \\
\hline Atoms & WP & Modes \\
\hline O2 & $6 i$ & $\mathrm{GM} 1+(2) \mathrm{GM} 3+(3) \mathrm{A} 2+(1) \mathbf{A 3}+(\mathbf{3})$ \\
\hline $\mathrm{O} 1 \mathrm{Cr} 1 \mathrm{~K} 1$ & $2 d$ & $\mathrm{GM} 1+(1) \mathrm{GM} 3+(1) \mathbf{A} 3+(\mathbf{1})$ \\
\hline $\mathrm{K} 2$ & $1 b$ & $\mathbf{A 3}+(\mathbf{1})$ \\
\hline
\end{tabular}

Figure 5. A screenshot from the AMPLIMODES program which shows the primary (in bold letters) and secondary modes which drive the triclinic-monoclinic transition and atoms in the unit cell which contribute to these modes.

high and low symmetry phases. Based on the known structure data it determines also the primary and secondary symmetry modes compatible with the determined sequence of phase transitions and calculates the amplitudes of the different symmetry-adapted distortions presented in the lowsymmetry phase as well as their polarization vectors. Our theory analysis was based on the experimental structure data of $\mathrm{K}_{3} \mathrm{Na}\left(\mathrm{CrO}_{4}\right)_{2}$ crystals determined in $[1,2]$ and summarized in the table.

The crystallographic unit cells of trigonal $(P \overline{3} m 1)$ and monoclinic $(C 2 / c)$ phases drawn with the VISUALIZE program of BSC are presented in Figs. 3 and 4, respectively.

The results of the analysis with the AMPLIMODES program are summarized below in Figs. 5 and 6.

From Fig. 6, we see that there are two possible intermediate phases: $C 2 / m(\# 12)$ and $P \overline{3} c 1(\# 165)$. However, the $\Gamma_{3}^{+}$ distortions $(0.0842 \AA)$ connected with the transition into the monoclinic intermediate phase $C 2 / m$ are 20 times larger than those connected with the transition into the trigonal intermediate phase $P \overline{3} c 1(0.0044 \AA)$. Therefore, the latter one can be neglected and excluded from our consideration. The distortion with $\Gamma_{3}^{+}$symmetry, which is responsible for the transition into monoclinic intermediate phase $C 2 / \mathrm{m}$, gives an essential contribution into the global distortion $(0.3788 \AA)$. Therefore, it appears to be observed in our experiments.

Summary of amplitudes

\begin{tabular}{|l|l|l|l|c|c|}
\hline$K$-vector & Irrep & \multicolumn{1}{|c|}{ Direction } & $\begin{array}{l}\text { Isotropy } \\
\text { subgroup }\end{array}$ & Dimension & Amplitude, $\AA$ \\
\hline$(0,0,0)$ & GM1+ & $(a)$ & $P-3 m 1(164)$ & 5 & 0.0199 \\
\hline$(0,0,0)$ & GM3+ & $(-0.500 a, 0.866 a)$ & $C 2 / m(12)$ & 6 & 0.0842 \\
\hline$(0,0,1 / 2)$ & A2+ & $(a)$ & $P-3 c 1(165)$ & 1 & 0.0044 \\
\hline$(0,0,1 / 2)$ & A3+ & $(-0.866 a,-0.500 a)$ & $C 2 / c(15)$ & 7 & 0.3687 \\
\hline
\end{tabular}

Global distortion: $0.3788 \AA$

Figure 6. A screenshot from the AMPLIMODES program which shows the amplitudes of the distortions connected with primary and secondary modes. 
The symmetry of the zone-edge soft mode above the phase transition temperature $210 \mathrm{~K}$ determined by the SYMMODES program [12] is $A_{2}^{+}$. (Our analysis was based on the atomic arrangement in the intermediate phase $C 2 / \mathrm{m}$ determined in our earlier paper [6].) This mode is not observed in the first-order Raman spectra (Figs. 1 and 2). The CORREL program of BCS [7] allowed us to determine that the $A_{2}^{+}$soft mode transforms into the $\Gamma_{1}^{+}$mode of the low-symmetry phase $C 2 / c$. Hence, the soft mode observed in our experiments (see Figs. 1 and 2) is the $\Gamma_{1}^{+}$one According to [15], this transition from the intermediate to the low symmetry phase is a non-ferroic one.

In the temperature region near the trigonal $(P \overline{3} m 1)$ monoclinic $(C 2 / m)$ phase transition $(239 \mathrm{~K})$, no soft modes were observed in the first-order Raman spectra. Hence no phonon modes are responsible for this transition. Our analysis (see Figs. 5 and 6) shows that this transition is driven by the active irreducible representation $\Gamma_{3}^{+}$. According to this representation the components of the strain tensor are transformed. Hence, the distortion $P \overline{3} m 1 \rightarrow C 2 / m$ with the $\Gamma_{3}^{+}$symmetry is due to the spontaneous strain. This phase transition is a proper ferroelastic one [15].

\section{Conclusion}

The results of Raman studies of the $\mathrm{K}_{3} \mathrm{Na}\left(\mathrm{CrO}_{4}\right)_{2}$ crystals within the low-frequency range of intermolecular (translational and librational) vibrations manifested the softening of the mode at the Brillouin zone boundary. It has been found that the frequency of the phonon mode $\left(33.5 \mathrm{~cm}^{-1}\right.$ at $8 \mathrm{~K}$ ) tends to zero when approaching to $T=210 \mathrm{~K}$. At temperatures $T>210 \mathrm{~K}$, this mode was not observed that evidences about the softening of the phonon mode at the Brillouin zone boundary. According to our group theory analysis, this mode has the $A_{2}^{+}$symmetry.

These results are consistent with our previous conclusions [6] about the existence of an intermediate monoclinic phase below $239 \mathrm{~K}$. The temperature interval of this phase found in the present studies is slightly narrower than in [6] that correlates with the results on the isostructural compound $\mathrm{K}_{3} \mathrm{Na}\left(\mathrm{SeO}_{4}\right)_{2}$. The transition from the highsymmetry trigonal phase into the monoclinic intermediate phase is driven by the $\Gamma_{3}^{+}$distortion which was shown to be a spontaneous strain.

The authors thank Professors M. Mączka and J. Hanuza for fruitful discussions.

\section{References}

[1] G. Madariaga, T. Breczewski. Acta. Cryst. C 46, 2019 (1990).

[2] J. Fabry, T. Breczewski, G. Madariaga. Acta. Cryst. B 50, 13 (1994).

[3] M. Kaczmarski, B. Mróz. Phys. Rev. B 57, 13589 (1998).

[4] A.R. Lim. J. Phys.: Condens. Matter 20, 135212 (2008).

[5] J. Díaz-Hernández, J.L. Mañes, M.J. Tello, A. López-Echarri, T. Breczewski, I. Ruiz-Larria. Phys. Rev. B 53, 14097 (1996).
[6] Yu.E. Kitaev, T.I. Maksimova, K. Hermanowicz, M. Mạczka, J. Hanuza. Opt. Spectroscopy 116, 858 (2014).

[7] M.I. Aroyo, A. Kirov, C. Capillas, J.M. Perez-Mato, A. Wondratschek. Acta Crystallogr. Sect. A 62, 115 (2006); www.cryst.ehu.es

[8] M.I. Aroyo, J.M. Perez-Mato, C. Capillas, E. Kroumova, S. Ivantchev, G. Madariaga, A. Kirov, A. Wondratschek. Z. Kristallogr. 221, 15 (2006).

[9] M.I. Aroyo, J.M. Perez-Mato, D. Orobengoa, E. Tasci, G. de la Flor, A. Kirov. Bulg. Chem. Commun. 43, 183 (2011).

[10] D. Orobengoa, C. Capillas, M.I. Aroyo, J.M. Perez-Mato. J. Appl. Cryst. A 42, 820 (2009).

[11] J.M. Perez-Mato, D. Orobengoa, M.I. Aroyo. Acta. Cryst. A 66, 558 (2010).

[12] C. Capillas, E. Kroumova, J.M. Perez-Mato, M.I. Aroyo, H.T. Stokes, D. Hatch. J. Appl. Cryst. 36, 953 (2003).

[13] A.S. Krylov, E.M. Kolesnikova, L.I. Isaenko, S.N. Krylova, A.N. Vtyurin. Cryst. Growth Design 14, 923 (2014).

[14] A.S. Krylov, E.M. Merkushova, A.N. Vtyurin, L.I. Isaenko. Phys. Solid State 54, 1275 (2012).

[15] H.T. Stokes, D.M. Hatch. Isotropy Subgroups of the 230 Crystallographic Space Groups World Scientific, Singapore (1988). 